\title{
Determination of Prevalence of Filariasis by Rapid Card Test in a Tertiary Care Centre of North India
}

\author{
Galhotra Shipra ${ }^{*}$, Prasad Amber ${ }^{2}$, Goyal Kapil ${ }^{3}$, Sehgal Rakesh $^{4}$, \\ Y. Pant ${ }^{4}$ and P. Tripathi ${ }^{4}$ \\ ${ }^{1}$ Department of Microbiology in GGS Medical College, Faridkot, Punjab, India \\ ${ }^{2}$ Department of Microbiology Ranchi Institute of Medical Sciences, Ranchi, Jharkhand, India \\ ${ }^{3}$ Department of Virology in Postgraduate Institute of Medical Education and Research \\ (PGIMER), Chandigarh, India \\ ${ }^{4}$ Department of Parasitology in Postgraduate Institute of Medical Education and Research \\ (PGIMER), Chandigarh, India \\ *Corresponding author
}

\section{A B S T R A C T}

The prevalence of lymphatic filariasis caused by Wuchereria bancrofti is very high in certain geographical regions and it is one of the neglected tropical diseases targeted for global elimination. So, there is an urgent need for the detection of circulating filarial

Keywords

ICT, Lymphatic filiariasis, TBF, Wuchereria bancrofti

Article Info

Accepted:

17 January 2018

Available Online:

10 February 2018 antigen by rapid immunoassay in Global Programme to Eliminate Lymphatic Filariasis (GPELF). This programme as a public health aspect focuses on mass drug delivery after community diagnosis. The Immunochromatographic card test (ICT) which does not detect early exposure to lymphatic filariasis (LF) but detects active infection is recommended test in current WHO guidelines. The mapping of LF by ICT card test in recent years has decreased the night mass blood surveys, time consuming preparations and examination of blood slides. This study investigated the use of rapid ICT test for the detection of filariasis. A total of 243 suspected cases of filariasis were tested by ICT and 38 were tested for thick blood film (TBF) examination. The positivity rate of filariasis was $8.6 \%$ by ICT card test and it was lower in females than males. None of the sample was positive by TBF. The ICT card test was positive in 5 cases which were negative by TBF. This study demonstrates that ICT card test because of its rapidity and simplicity justifies its use for the detection of filariasis. This test should be applied in field studies and would be helpful in GPELF.

\section{Introduction}

Lymphatic filariasis (LF) is a disease that spreads by mosquito vector and it can be presented as severe lymphoedema, hydrocele and elephantiasis (Taylor et al., 2010). It is a chronic debilitating disease mainly caused by the parasites Wuchereria bancrofti and Brugia malayi. The transmission of disease is by various species of mosquitoes from the genera Anopheles, Aedes, Culex, Mansonia and Ochlerotatus. The list of various infectious 
diseases which are targeted for global elimination also includes filariasis (Task Force for Global Health, 2014). The disease affects about 128 million people in more than seventy countries with an estimated 1.1 billion are threatened by LF (Addiss and Brady, 2007).

The disease persists as second leading cause of disability and debility worldwide, inflicting serious public health and socio-economic burden in endemic communities. Mass drug administration (MDA) following community diagnosis by WHO has played its role in the interruption of transmission of this disease (Omudu and Ochoga, 2011). For successful elimination of LF and to effectively target MDA programs accurate mapping of endemic regions is required (Foo et al., 2011). The filariasis control programme should be properly planned, executed and monitored with active participation of public health system for the prevention of filariasis (Braga et al., 2003). Essential requirement for the control of filariasis is reliable and accurate identification of infection and transmission by rapid diagnostic test which is to be accomplished (Alli et al., 2001).

The use of thick blood film (TBF), a usual diagnostic method, for the diagnosis of Bancroftian filariasis, has many limitations. Firstly, people generally give refusals for the collection of blood during night and there is an increased cost of this test. Secondly, there is relatively low (3-6\%). sensitivity of this method. Also, people with low level of microfilaraemia and amicrofilaria are usually undetected by conventional microscopy (Foo et al., 2011). This lack of diagnosis leads to major irreversible lymphatic damages in patients having filariasis. Immuno chromatographic test (ICT), a rapid format test kit based on detection of circulating filarial antigen using anti Dirofiliaria immitis monoclonal antibody AD 12.1 in blood samples, for diagnosis of bancroftian filariasis, has various advantages (Braga et al., 2003). It can conveniently be performed on finger prick blood samples and time to detection is less.

The lateral-flow Immunochromatographic test for the detection of adult-specific circulating filarial antigen (CFA) is playing its role in transmission assessment surveys (TAS) for post-MDA surveillance under WHO guidelines (Steel et al., 2013). These significant features make the whole blood ICT card test a ready diagnostic tool due to its ease of use (Omudu and Ochoga, 2011). It is extremely useful in detecting active Wuchereria bancrofti infection, but the limitation of the ICT is that it cannot detect infection prior to the development of adult parasites (Alli et al., 2001).

The purpose of this study is to determine prevalence of filariasis by detecting circulating filarial antigen by rapid ICT (Binax now) and microfilaria in Giemsa stained blood smears among suspected cases of filariasis.

\section{Materials and Methods}

Study Site: Department of Medical Parasitology

Study Period: April 2011 to 2014

Type of study: Retrospective study

Study subjects: 243 suspected cases of filariasis

Tests performed: ICT: 243; Microscopy: 38

This is a retrospective study in which a total of 243 suspected cases of filariasis were subjected to Immunochromatographic test for the detection of Wuchereria bancrofti antigen and 38 peripheral blood smears were analyzed for Mf by microscopy as a part of routine testing for filariasis in the Department of Medical Parasitology, PGIMER, Chandigarh from April 2011 to March 2014. 


\section{Diagnostic techniques for Lymphatic Filariasis}

Thick blood film: These blood slides detects microfilariae which is the larval form of Wuchereria bancrofti through finger prick blood samples collected during night to increase the detection of microfilariae present in peripheral blood. The microfilaraemia load in the blood stream must be enough for the detection of microfilariae (mf) as it is the definitive method for the diagnosis of filariasis. Microfilariae density should be $20 \mathrm{mf} / \mathrm{ml}$ in blood for the detection of microfilariae in thick blood film (Nutman, 2000).

A thick blood film is allowed to dry in a dust free area. The thickness of the film should be such as to allow newsprint to be read or the hands of a wrist watch to be seen through the dry preparation. Once the film is dry, it should be dehaemoglobinized in distilled water in vertical position. The film is fixed by covering it with absolute methyl alcohol for $3 \mathrm{~min}$, stained with haematoxylin (Carrazi method) and examined microscopically.

ICT card test (AMRAD ICT, New South Wales, and Australia): The test detects filarial antigen in the blood of infected humans by using paired antibodies (polyclonal and monoclonal antibodies). The characterization of antigen (or antigens) bound by monoclonal antibody (AD12) have not been entirely established, but they seem to be found in all stages of Wuchereria bancrofti (Nutman, 2000). The cards were stored in a refrigerator at $8^{\circ} \mathrm{C}$. The reading and interpretation of tests were done in the parasitology laboratory by the technical staff. The reading and interpretation of the results followed the manufacturer's instructions. Results and Discussion
The number of males suspected to be suffering from lymphatic filariasis was more as compared to females (Table 1).

The number of adults (90\%) was more as compared to children (10\%) in this study (Table 2).

The overall positivity in the present study was $8.6 \%$ by ICT and none of the sample tested positive for Mf by microscopy (Table 3). There was increase in percentage positivity with ICT card tests yearly but increase was not so much (Figure 1).

The assessment of potential ongoing transmission of the filarial parasite that causes lymphatic filariasis (LF) can be done by highly sensitive and specific test which is one of the immediate requirements in the post -MDA world. This test should be rapid and cost effective at the point of care and in centralized laboratories where large no of samples are to be performed. Current WHO guidelines for assessment of Wuchereria bancrofti transmission is detection of circulating filarial antigen (CFA) by ICT method (Alli et al., 2001).

Filariasis prevalence was $8.6 \%$ in this study which is almost similar (10.1\%) to previously published study by (Grave et al., 2013). In Sierra region lymphatic prevalence was found to be $21 \%$ which is higher than this study (Koorma et al., 2012). According to global programme of elimination of lymphatic prevalence (GPELF) this indicates the need for mass drug administration for suspected lymphatic filariasis patients because prevalence is higher than $1 \%$ which is the recommendation of this programme.

Table.1 No. of samples examined (\%)

\begin{tabular}{|l|l|l|l|}
\hline Test & Total & Male & Female \\
\hline Thick Blood Film & 38 & $26(68 \%)$ & $12(32 \%)$ \\
\hline ICT & 243 & $160(66 \%)$ & $83(34 \%)$ \\
\hline
\end{tabular}


Table. 2 Comparison in adults and children

\begin{tabular}{|l|l|l|}
\hline Total & Adult & Children \\
\hline 243 & $219(90 \%)$ & $24(10 \%)$ \\
\hline Positive (21) & $16(76 \%)$ & $5(24 \%)$ \\
\hline
\end{tabular}

Table.3 No. of positives (\%) classified according to sex and diagnostic methods

\begin{tabular}{|l|l|l||l|}
\hline Test & Total & Male & Female \\
\hline Thick Blood Film & $0 / 38$ & 0 & 0 \\
\hline ICT & $21 / 243(8.6 \%)$ & $14 / 243(5.8 \%)$ & $7 / 243(2.8 \%)$ \\
\hline
\end{tabular}

Table.4 Microscopy negative and ICT positive samples

\begin{tabular}{|c|c|c|}
\hline Total & Mf & ICT \\
\hline 38 & 0 & $5(13.15 \%)$ \\
\hline
\end{tabular}

Fig.1 Year wise data

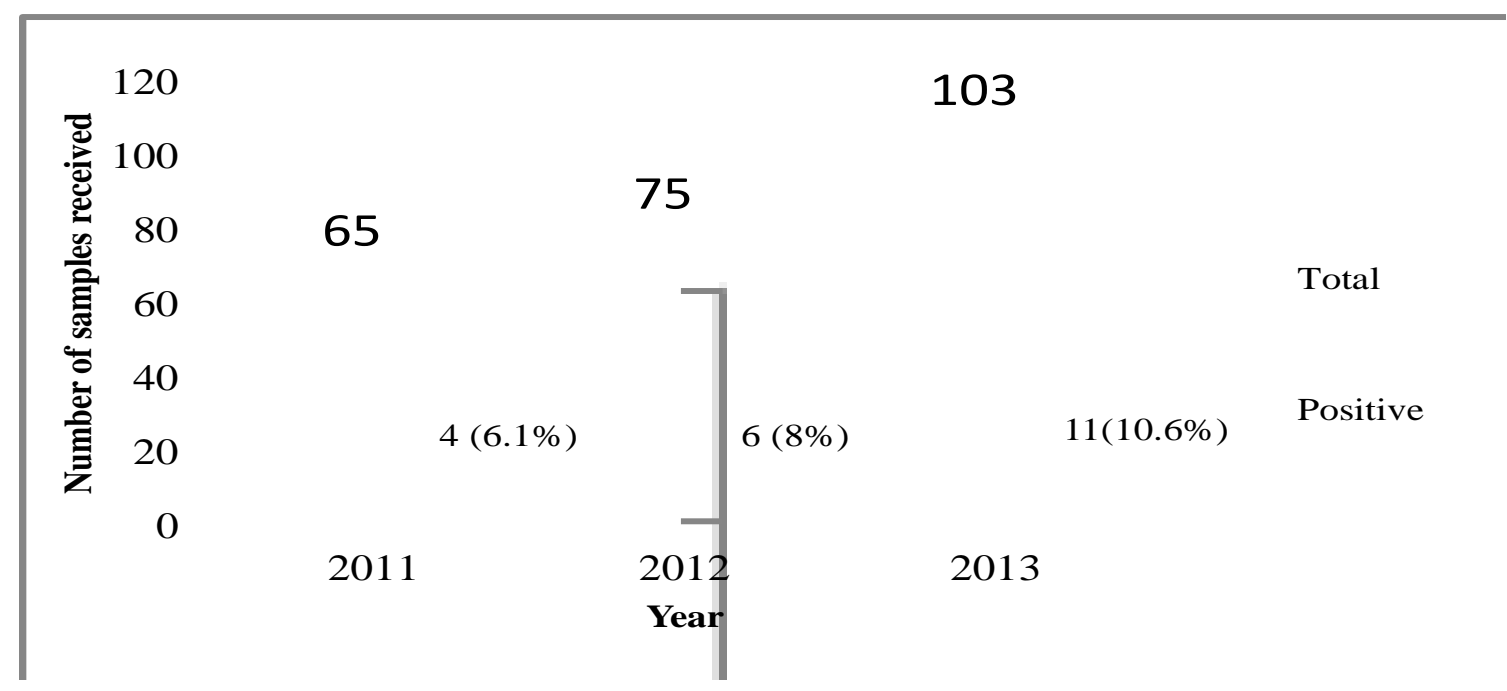

The prevalence of LF is higher in males than females especially among adults in many endemic areas (Brabin, 1990; Mwakitalu et al., 2013) Similar finding has been found in this study.

The prevalence of microfilaraemia by thick blood film (TBF) was three to five times lower than the antigenemia detected by ICT card tests as ICT detects antigen released by the adult worm of Wuchereria bancrofti
(Bhumiratana et al., 1999) In this study none of the samples were positive by microscopy but ICT positivity was $8.6 \%$. In another study by Braga et al., prevalence of filariasis by TBF was $6.08 \%$ and by ICT was $31 \%$. So this study also showed good results of ICT card test as compared to thick blood film examination for microfilariae.

Conclusions: The result of this study suggests that the ICT is rapid format antigen for the 
detection of filariasis and thus helps in the effective management of patients suffering from filariasis and preventing recurrence in the endemic area.

\section{References}

Addiss DG, and Brady MA. 2007. Morbidity management in the global programme to eliminate lymphatic filariasis: a review of scientific literature. Filarial Journal 6:21-33.

Alli R et al., 2001. Evaluation of Sevafilachek immunoassays and rapid ICT filariasis test for detection of Bancroftian filariasis. Indian J Clin Biochem 16(2):207-10.

Bhumiratana A et al., 1999. Field trial of the ICT filariasis for diagnosis of Wuchereria bancrofti infections in an endemic population of Thailand. Southeast Asian J Trop Med Public Health 30:562-8.

Brabin L. 1990. Sex differentials in susceptibilty to lymphatic filariasis and and implications for maternal child immunity. Epidemiol Infect 105:335-53

Braga C et al., 2003. Field evaluation of the whole blood immunochromatographic test for rapid Bancroftian filariasis diagnosis in the northeast of Brazil. Rev Inst Med Trop S. Paulo 45(3):125-9.

Foo PK et al., 2011. High prevalence of Wuchereria Bancrofti as detected by immunochromatographic card testing in five districts of Orissa, India, previously considered to be non-endemic. Trans $\mathrm{R}$ Trop Med Hyg 105(2):109-14.
Graves PM et al., 2013. Lymphatic filariasis in Papua New Guinea: Distribution at district level and impact of mass drug administration, 1980-2011. Parasites and Vectors 6:7.

Koorma JB et al., 2012. Lymphatic filariasis mapping by immunochromatographic tests card and baseline microfiliaria survey prior to mass drug administration in Sierra Leone. Parasites and Vectors 5:10.

Mwakitalu ME et al., 2013. Urban lymphatic filariasis in the metropolis of Dar es Salaam, Tanzania. Parasites and Vectors 6:286

Nutman T.B. 2000. Lymphatic filariasis. London, Imperial College Press 127150.

Omudu AE, and Ochoga JO. 2011. Clinical epidemiology of lymphatic filariasis and community practices and perceptions amongst the Ado people of Benue State, Nigeria. Afr J Infect Dis 5:47-53.

Steel C et al., 2013. Rapid Wuchereria bancrofti-specific antigen wb123-based igg4 immunoassays as tools for surveillance following mass drug administration programs on lymphatic filariasis. Clin Vaccine Immunol 20:1155-61.

Task Force for Global Health: Meeting of the international task force for disease eradication, January 2014. Wkly Epidemiol Rec 2014; 89:153-60.

Taylor MJ et al., 2010. Lymphatic filariasis and onchocerciasis. Lancet 376:117585 .

\section{How to cite this article:}

Galhotra Shipra, Prasad Amber, Goyal Kapil, Sehgal Rakesh, Y. Pant and Tripathi, P. 2018. Determination of Prevalence of Filariasis by Rapid Card Test in a Tertiary Care Centre of North India. Int.J.Curr.Microbiol.App.Sci. 7(02): 1857-1861.

doi: https://doi.org/10.20546/ijcmas.2018.702.223 